\title{
The Modified WHO Analgesic Ladder: Is It Appropriate for Chronic Non-Cancer Pain?
}

This article was published in the following Dove Press journal:

Journal of Pain Research

\author{
Juan Yang ${ }^{1,2}$ \\ Brent A Bauer (iD) ${ }^{2}$ \\ Dietlind L Wahner- \\ Roedler (iD ${ }^{2}$ \\ Tony Y Chon $\mathbb{D D}^{2}$ \\ Lizu Xiao'
}

'Department of Pain Medicine, Shenzhen Nanshan People's Hospital, Shenzhen 518052, People's Republic of China; ${ }^{2}$ Division of General Internal Medicine, Mayo Clinic, Rochester, MN 55905, USA

Correspondence: Lizu Xiao Department of Pain Medicine, Shenzhen Nanshan People's Hospital, NO. 89,

Taoyuan Avenue, Nanshan District, Shenzhen 518052, People's Republic of China

Tel +86 755-26553 III

Email nsyyjoe@live.cn
Introduction: From 1986, the World Health Organization (WHO) analgesic ladder has been used as the simple and valuable pain-relieving guidance in the pharmaceutical pain management, however, with the development of medical history, notions about pain physiology and pain management have already updated. Is the analgesic ladder still appropriate for chronic non-cancer pain (CNCP) patients? This study aims to analyse the current usage of the analgesic ladder in patients with CNCP by evaluating previously published pertinent studies.

Methods: Literature published in English from January 1980 to April 2019 and cited on PubMed database was included. Analysis on the analgesic ladder, current status of CNCP management, and a new revised ladder model were developed based on relevant literature.

Results: The WHO analgesic ladder for cancer pain is not appropriate for current $\mathrm{CNCP}$ management. It is revised into a four-step ladder: the integrative therapies being adopted at each step for reducing or even stopping the use of opioid analgesics; interventional therapies being considered as step 3 before upgrading to strong opioids if non-opioids and weak opioids failed in CNCP management.

Discussion: A simple and valuable guideline in past years, the WHO analgesic ladder is inappropriate for the current use of $\mathrm{CNCP}$ control. A revised four-step analgesic ladder aligned with integrative medicine principles and minimally invasive interventions is recommended for control of CNCP.

Keywords: analgesic ladder, chronic pain, chronic non-cancer pain, opioid, pain management

\section{Introduction}

In the past two decades, an opioid epidemic has raged worldwide, with opioid abuse becoming a very common social problem in developed countries, especially in the United States, with the rate of opioid overdoses tripling since $2000 .{ }^{1}$ The origins of the "opioid crisis" are complex and multifaceted, including inappropriate prescribing, ${ }^{2}$ lack of knowledge regarding adverse events associated with long-term use, opioid misuse, abuse and addiction. ${ }^{3}$ A key factor also appears to be the prescription of opioids for chronic non-cancer pain (CNCP) for long-term use. ${ }^{4}$

Chronic pain is defined by the International Association for the Study of Pain (IASP) as persistent or recurrent pain lasting more than 3 months or beyond the normal tissue healing period. ${ }^{5} \mathrm{CNCP}$ refers to any chronic pain that is not due to a malignancy. CNCP can be classified as neuropathic pain or nociceptive pain. ${ }^{6}$ It is a globally encountered problem in the healthcare field and often triggers physiological, social, and psychological responses, which may consequently seriously harm people's health, functioning, and well-being. ${ }^{7}$ It is a significant health concern with negative 
consequences for individuals, families, and society as a whole. Because it is a multidimensional morbid condition with both objective and subjective characteristics, CNCP is difficult to assess and manage in clinical practice. However, despite limited scientific evidence to suggest efficacy, the use of opioid medication for $\mathrm{CNCP}$ has been increasing substantially. ${ }^{8,9}$ Though less than $5 \%$ of the global population, Americans consume $80 \%$ of the world's opioid prescriptions. ${ }^{10}$ In 2009 a multidisciplinary panel from The American Pain Society (APS) and the American Academy of Pain Medicine (AAPM) conducted a systematic review and formulated recommendations of chronic opioid therapy for CNCP concluding that chronic opioid therapy was recommended as a possibly effective therapy for carefully selected and monitored CNCP patients. ${ }^{11}$ Accounting for the prevalence of CNCP and its disabling features, opioids have already been the most prescribed medication in the USA. ${ }^{12}$ About $10-30 \%$ of CNCP patients have comorbid substance use and patients with substance use history who are prescribed opioid therapy are more likely to misuse. ${ }^{13}$ In 2018 , chronic opioid therapy was definitely recommended to CNCP patients with proven medical necessity and stable pain and function improvement, independently or jointly with other treatment modalities. ${ }^{14}$

However, the goals of pain management are to reduce pain and improve daily function while monitoring for adverse effects. ${ }^{15}$ Opioid analgesics are a double-edged sword: while they are remarkable drugs that can provide a modest improvement in pain and function for a wide range of CNCP, they may also lead to opioid-induced neurotoxicity, risks of tolerance and physiological and psychological dependence. The role of opioid analgesics in the long-term management of $\mathrm{CNCP}$ syndromes remains controversial due to insufficient evidence of efficacy and increasing reports of adverse effects. ${ }^{16,17}$ The Centers for Disease Control and Prevention (CDC) has reported that in the United State, approximately 130 persons die each day from opioid overdoses, with $68 \%$ of the drug overdose deaths in 2017 involving an opioid medication. These alarming statistics indicate that this current crisis has created a major public health challenge with unprecedented rates of significant morbidities, health-related complications, and even unintentional overdose deaths. It is a nationwide public-health emergency requiring national recognition.

Even though multiple guidelines have been published for the long-term use of opioid therapy in $\mathrm{CNCP},{ }^{14,18-21}$ at the present time, there is no guideline specifically addressing the usage of the WHO analgesic ladder. It is likely that the ladder will continue to be used for CNCP management, but is this appropriate?

In this literature-based brief review, we explore the correlation between the WHO analgesic ladder and the opioid crisis, and the current status of CNCP management. Furthermore, in light of the controversial issue of the current WHO analgesic ladder addressing the opioid epidemic, we discuss the importance of revising the WHO analgesic ladder and call for adoption of a revised fourstep analgesic ladder concept. We believe it will not only remind medical providers to think and act comprehensively in seeking solutions, but it also aligns the treatment goals with integrative medicine therapies and minimally invasive interventions in dealing with CNCP.

\section{Methods}

Literature published in English from January 1980 through April 2019 and cited on PubMed database was searched using the terms analgesic ladder, chronic pain, chronic noncancer pain, opioid, pain management. Given the broad historical context relevant to the topic, this brief review only addresses the most relevant and recent articles.

\section{Results}

\section{WHO Analgesic Ladder and Opioid Crisis}

The World Health Organization (WHO) first released a document addressing cancer pain relief in 1986, which stipulated a Three-step analgesic ladder as the guideline for developing treatment plans for cancer pain. ${ }^{22}$ It was revised in $1996 .{ }^{23}$ Prior to the guideline's release, many patients suffered from unnecessary pain due to the stigma and fear associated with both the prescription of opioids and their utilization. ${ }^{24}$ The WHO analgesic ladder specifies treatment on pain intensity, from simple analgesics for mild pain to opioid analgesics for moderate and severe pain. Its three steps are: Step 1 Non-opioid plus optional adjuvant analgesics for mild pain; Step 2 Weak opioid plus non-opioid and adjuvant analgesics for mild to moderate pain; Step 3 Strong opioid plus non-opioid and adjuvant analgesics for moderate to severe pain. It is advised to move up one step when there is persistent pain. In case of toxicity or severe adverse effects, providers are advised to either reduce medication doses or move down one step. The ladder provides five simple recommendations for the usage of analgesics: by mouth, by clock, by ladder, by individual and attention to the detail. Just two years 
following its release, it was already validated in $80-90 \%$ of cases. ${ }^{25}$ The stepwise approach had tremendous value when it was introduced for its conservative and simple principles for pain management, which could be applied everywhere in the world, even in those underdeveloped countries with fewer pain management specialists. It has been of significant benefit for the control of pain worldwide. Until now, this guideline has remained applicable, not only in cancer pain management but also for acute pain and chronic pain requiring analgesics. ${ }^{26}$

The three-step ladder for cancer pain has also been commonly employed in CNCP, which very likely contributed to opioid analgesic overuse and escalation. ${ }^{26,27}$ For ensuring patients with safer and more effective chronic pain management, the CDC in 2016 released a guideline regarding the prescribing of opioid pain medication for adult patients with CNCP in the primary care setting. The concept of pain relief as a fundamental human right acknowledges access of patients to essential medicines, including opioid medications for the management of pain. According to the WHO analgesic ladder, the provider prescribes opioid analgesics to patients based on the patient's report of how serious the pain is. In the United States, the top 4 opioids prescribers are physicians in family practice, internal medicine, and nurse practitioners, and physician's assistants, with the majority of them not specifically trained in pain control to a significant degree. ${ }^{14,28-30}$ It has been shown that chronic pain patients obtain opioid medication easily from physicians. ${ }^{2,31}$ A study in 2014 reported that patients received opioid analgesic prescriptions from two providers in $34.6 \%$ of cases, $14.2 \%$ from three providers, and $11.9 \%$ from four or more prescribers. ${ }^{32}$ There were 240 million opioid prescriptions dispensed in 2015 in the United States, almost one for every adult in the general population. ${ }^{33}$ Prescriptions thus have probably been a major contribution to the issues of opioid addiction and overdose deaths. ${ }^{34}$ Furthermore, some physicians prescribe more than one-month of opioid medication to patients, ${ }^{35}$ while too many patients leave hospitals with bottles of unnecessary opioid analgesics. ${ }^{2}$ There are many cases of nonmedical users of opioids obtaining them from their relatives or friends, just for experimentation or for "getting high". ${ }^{36}$ United States poison control centers reported 188,468 prescription opioid exposures among children aged $<20$ years old from 2000 to $2015 .^{37}$ More than 2,000,000 people are suffering from prescription opioidrelated substance abuse currently, ${ }^{38}$ which highlights the fact that this is a particularly devastating problem in public health. Therefore, how to prescribe the opioid analgesics appropriately can be particularly challenging and stressful for primary care providers. Changes in opioid prescription policies should include sufficient training, including the applicability of analgesic ladder, which is a valuable guideline addressing both cancer and non-cancer pain.

\section{Is WHO Analgesic Ladder Appropriate to CNCP?}

Thirty-three years are not a short time in the medical field, notions about pain physiology and pain management have changed considerably. First, there is greater recognition of the various types of pain e.g. nociceptive, neuropathic and et $\mathrm{al}^{39}$ as well as the need of tailoring treatment decisions accordingly. Second, because of the complexity of pain, a number of new pain management strategies, new opioid analgesics and other novel pharmaceuticals emerged. Thus, attempting to manage pain with only the original analgesic ladder guideline is inappropriate. It is now necessary to incorporate multimodal and multidisciplinary approaches into the WHO Three-step ladder. The current CDC guidelines conclude that opioid analgesics are not first-line or routine therapy for chronic pain management, with nonpharmacological therapy and non-opioid pharmacological treatment being preferred for chronic pain. ${ }^{40}$ This guidance aims to reduce risk factors associated with long-term opioid usage and improve safety and effectiveness of pain management. The 2018 guidelines published by American Society of Interventional Pain Physicians (ASIPP) have already recommended non-opioid pharmacological and, nonpharmacological treatments, such as acetaminophen or other NSAIDs, pregabalin, integrative medicine therapies and multiple minimal invasive techniques, etc. ${ }^{14}$ Interventional techniques, such as epidural injections, percutaneous epidural adhesiolysis, and neuromodulation etc., can alter pain management and outcome measurements of many $\mathrm{CNCP}$ patients. Minimally invasive therapies have been already definitely recommended in practice guidelines for CNCP management. ${ }^{41}$ Integrative medicine, a holistic model of care which aims to selectively combine the exclusively evidence-based complementary and alternative therapies into conventional medicine, ${ }^{42}$ has risen in popularity in recent years among patients and medical providers for the relief of chronic or multifactorial conditions such as CNCP. ${ }^{43}$ It is a combination of medical treatments such as acupuncture, massage relaxation techniques, healing touch, etc. ${ }^{44,45}$ Clinical studies have shown that acupuncture is 
a reasonable effective option for multiple chronic pain conditions. ${ }^{46}$ There is a rising interest in using acupuncture for CNCP instead of reliance on opioid analgesics. ${ }^{43}$ The substantial basic knowledge development on pain and its therapies has led to the CNCP guidelines being updated, ${ }^{14,18-21,47}$ while the WHO three-step analgesic ladder has remained unchanged.

The WHO analgesic Ladder is not appropriate for current $\mathrm{CNCP}$ management and many commentators have noted that this current version has some limitations and controversies as a guide for current pain management. ${ }^{27}$ Some have suggested an analgesic "pyramid" which incorporates modern approaches. ${ }^{48}$ Some extended the concept of pain management from an up-down the linear pharmacological ladder to a broader three-dimensional platform. ${ }^{49}$ Some changed it into a four bidirectional step - "trolley analgesic model" - which postulates the use of the invasive procedures and adopted tailored therapies with dynamic multimodal pain management approaches. ${ }^{50}$ Others have called for a four-step ladder including pain control ${ }^{48}$ or a five-step ladder to include anesthetic intervention therapies and opioid switching. ${ }^{51}$

\section{Revised WHO Analgesic Ladder}

Better pain management is a major component in addressing the current opioid crisis. As a chronic condition involving physiological, social, and subjective aspects, CNCP should not be treated with pharmacological agents alone. Perhaps the major deficiency in the current ladder is that it does only emphasize the pharmacological treatment for pain, instead of adequately addressing that importance of nonpharmacological therapy and non-opioid therapy. The nonpharmacological and non-opioid therapies are the first-line therapeutic modalities recommended by the 2016 CDC Guideline for Prescribing Opioids for Chronic Pain. Our adaption of the analgesic ladder for CNCP offered here is based on the same principle. First, although integrative therapies are not shown on the original Three-step analgesic ladder diagram, they can be considered at each step of the revised ladder. About $27 \sim 60 \%$ of chronic pain patients use integrative medicine treatments. ${ }^{7}$ In a brief critical review of integrative medical therapies including acupuncture, massage, yoga, relaxation, tai chi, and spinal manipulation for chronic pain management, positive evidence was found. Especially strong evidence was noted for acupuncture as a complementary treatment for chronic pain which could reduce the dosage of opioid analgesics or even potentially relieve chronic pain without reliance on opioids. ${ }^{52}$ Second, minimally invasive interventions should be considered in step 3 when the nonopioids and weak opioids have failed to control the pain. This revision modifies the step 3 to include minimally invasive interventional therapies, such as nerve block, radiofrequency, spinal cord stimulation, spinal (epidural and subarachnoid) administration of local anesthetics, surgical intervention, and disc decompression and so on. Patients may try minimally invasive interventional therapies after they fail treatment with non-opioids and weak opioid medication and integrative therapy. Neuromodulation spinal cord stimulation can stabilize or decrease opioid usage by improving outcomes in complex chronic pain. ${ }^{53}$ As the procedure is minimally invasive and can help to figure out the problem if the diagnosis is not very clear, such as the discography therapy which is a golden diagnostic method for the discogenic pain. ${ }^{54}$ Finally, if the above modalities fail, strong opioid medication should be prescribed to the CNCP patient as "the last resort", i.e. step 4. The newly adapted version of the WHO analgesic ladder is shown in the following (see Figure 1).

Furthermore, due to various factors, such as economic, social and cultural determinants, etc., different countries may apply the guideline differently. Compared to Europe and USA, the consumption of opioid analgesics has been reported to be very low in some Asian countries such as China, India, and Thailand. ${ }^{55}$ In China, the average morphine equivalent (MEs) in the human development index (HDI) was $2.86 \mathrm{mg}$ per capita, much lower than the average level of the top 20 countries with $216.7 \mathrm{mg}$ per capita in $2010 .^{56}$ The major barriers for opioid prescribing came from patients' over-concern about opioids addiction, their reluctance to report their pain control condition and resistance to using opioids analgesics except for intolerable pain. ${ }^{57}$ Patients with moderate or severe pain received mild opioids in $44.72 \%$ and $45.83 \%$, respectively, and among them, only $34.72 \%$ and $27.08 \%$ received strong opioids. More than half of the patients refused opioids analgesics because of concerns regarding the potential addiction and adverse effects. ${ }^{58}$ Chinese annual defined opioid analgesics daily doses and morphine equivalents are lower than the global average. ${ }^{59}$ The lower consumption of opioid analgesics in China could also be attributed to two other factors. One is that numerous integrative medicine therapies such as acupuncture, cupping, Qigong and Chinese herbs, etc., are readily accessible and most can be performed for pain-relieving on an outpatient basis. ${ }^{60,61}$ Integrative Medicine offers people a variety of alternative treatments for pain management. The other factor is that the Chinese government and health organizations have made 


\section{Chronic non-cancer pain (CNCP)}

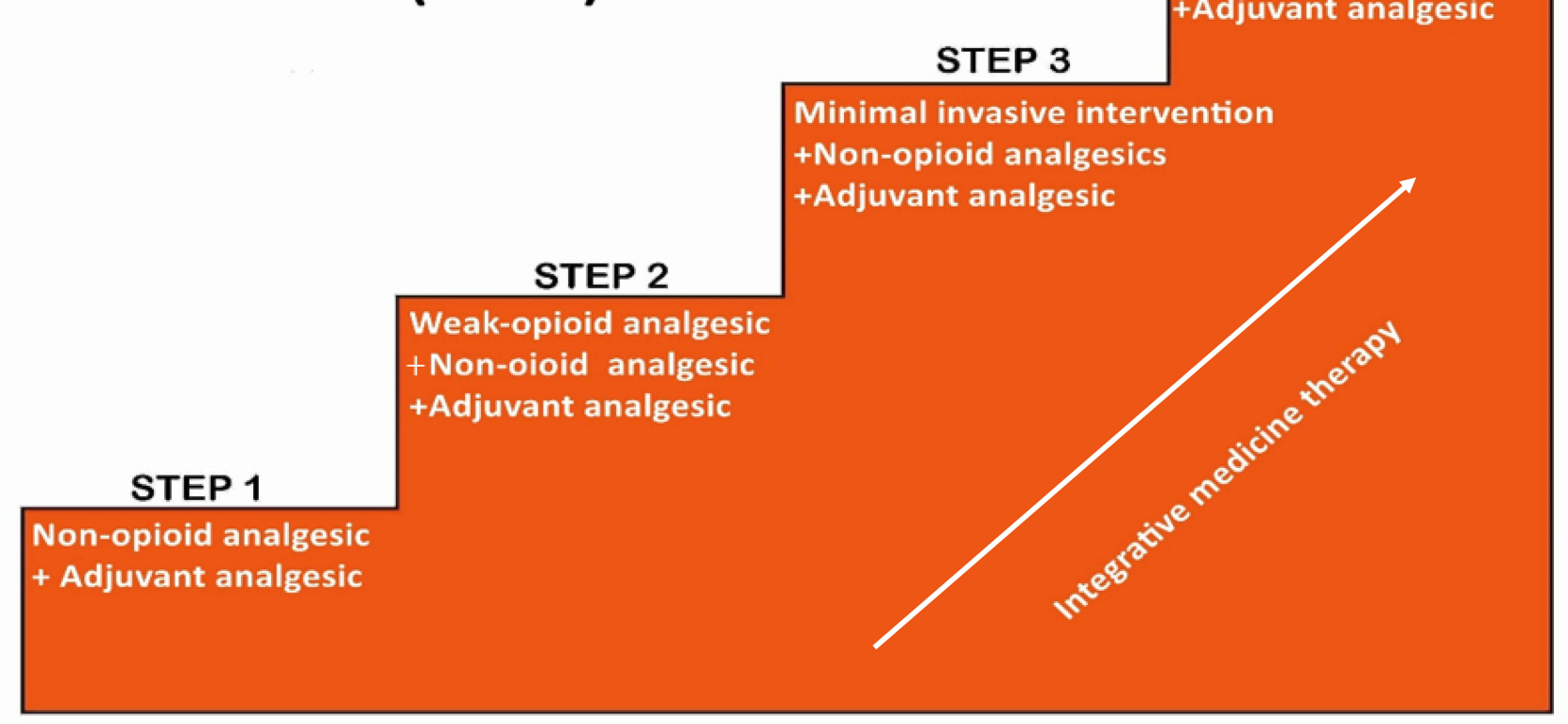

Figure I A generalized representation of a four-step analgesics ladder. Such four-step ladder, as opposed to the 1986 "ladder", reflects the advances in nonopioid modalities application for better pain relieving. The integrative medicine therapies can be adopted in each step for reducing or even stopping the use of analgesics to all types of pains. If the non-opioids and weak opioids failed, minimally invasive interventions in step 3 can be recommended before upgrading to strong opioids.

great efforts to address drug control. The government issued regulations on the administration of anesthetic drugs and psychotropic drugs, ${ }^{62}$ which regulates the administration of opioids, ensures their legitimate medical utilization, and limits illegal abuse. In 2007, the Ministry of Chinese Health set up a "Department of Pain Medicine" in the List of Medical Subjects for Medical Institutions and definitely noticed the main management scope of the Pain Medicine Department is: Diagnosis and treatment of chronic pain. The department belongs to the surgical category. Department staff composition includes licensed providers in anesthesiology, orthopedics, neurology, neurosurgery, rheumatology, oncology or rehabilitation medicine, and other physicians with experience skills in pain management. ${ }^{63}$ This release initially separated the Pain Medicine Department from Anesthesiology and legally defined the multidisciplinary composition of the practitioners in the pain department and the multimodalities of diagnosis and treatment. The Pain Management providers are privileged to adopt a series of minimally invasive interventions for chronic pain control, which significantly reduce dependence on pharmacological drugs, especially opioids in their clinical practice. When the non-opioid medications and weak opioid analgesics fail, medical professionals will be guided to minimally interventional treatments before upgrading to strong opioids. These positive findings also keep in line with our revised analgesic ladder model.

\section{Conclusion and Future Action}

In conclusion, the current WHO analgesic ladder, which was designed for cancer pain management, is a simple and valuable guideline in past years, but it seems that such a simple approach is inappropriate for the current updated pain management situation, especially the control of CNCP. We revised the original analgesic ladder into a four-step ladder. The integrative therapies can be adopted at each step for reducing or even stopping the use of opioid analgesics. If non-opioids and weak opioids failed, interventional therapies can be considered as step 3 before upgrading to strong opioids. Modification of the WHO analgesic ladder is likely to open a new path toward more effective pain management as an interdisciplinary challenge. As a result, we suggest adoption of this revised four-step analgesic ladder concept into clinical practice. The hope is that this will both remind medical providers to think and act comprehensively when seeking pain management solutions, and align the treatment goals with 
integrative medicine and minimally invasive interventional therapy in dealing with CNCP. Conducting pain management in a holistic manner, which integrates multidisciplinary and multimodality therapies for patients who are suffering from pain will be a key factor in mitigating the opioid epidemic.

\section{Acknowledgments}

This study was supported by Sanming Project of Medicine in Shenzhen, Shenzhen Nanshan People's Hospital, China. The opinions and assertions contained herein are the private views of the authors.

\section{Disclosure}

The authors have indicated that they have no conflicts of interest with regard to the content of this article.

\section{References}

1. Rudd RA, Aleshire N, Zibbell JE, Matthew Gladden R. Increases in drug and opioid overdose deaths-United States, 2000-2014. Am $J$ Transplant. 2016;16(4):1323-1327. doi:10.1111/ajt.13776

2. Makary MA, Overton HN, Wang P. Overprescribing is major contributor to opioid crisis. BMJ (Clinical Research Ed). 2017;359: j4792. doi:10.1136/bmj.j4792

3. Vadivelu N, Kai AM, Kodumudi V, Sramcik J, Kaye AD. The opioid crisis: a comprehensive overview. Curr Pain Headache Rep. 2018;22 (3):16. doi:10.1007/s11916-018-0670-z

4. Häuser W, Schug S, Furlan AD. The opioid epidemic and national guidelines for opioid therapy for chronic noncancer pain: a perspective from different continents. Pain Rep. 2017;2:3. doi:10.1097/PR9.00000000 00000599

5. Merskey HE. Classification of chronic pain: descriptions of chronic pain syndromes and definitions of pain terms. Pain. 1986.

6. Els C, Jackson TD, Hagtvedt R, et al. High-dose opioids for chronic non-cancer pain: an overview of cochrane reviews. Cochrane Database Syst Rev. 2017;10:Cd012299.

7. Treatment CfSA. Managing chronic pain in adults with or in recovery from substance use disorders. 2012. doi:10.1094/PDIS-11-11-0999PDN

8. Turner JA, Shortreed SM, Saunders KW, LeResche L, Von Korff M. Association of levels of opioid use with pain and activity interference among patients initiating chronic opioid therapy: a longitudinal study. Pain. 2016;157(4):849-857. doi:10.1097/j.pain.0000000000000452

9. Reinecke H, Weber C, Lange K, Simon M, Stein C, Sorgatz H. Analgesic efficacy of opioids in chronic pain: recent meta-analyses. $\mathrm{Br}$ J Pharmacol. 2015;172(2):324-333. doi:10.1111/bph.2015.172.issue-2

10. Manchikanti L, Singh A. Therapeutic opioids: a ten-year perspective on the complexities and complications of the escalating use, abuse, and nonmedical use of opioids. Pain Physician. 2008;11(2 Suppl):S63-S88.

11. Chou R, Fanciullo GJ, Fine PG, et al. Clinical guidelines for the use of chronic opioid therapy in chronic noncancer pain. J Pain. 2009;10 (2):113-130. doi:10.1016/j.jpain.2008.10.008

12. Volkow ND, McLellan AT. Opioid abuse in chronic pain-misconceptions and mitigation strategies. $N$ Engl $J$ Med. 2016;374 (13):1253-1263. doi:10.1056/NEJMra1507771

13. Hurstak EE, Kushel M, Chang J, et al. The risks of opioid treatment: perspectives of primary care practitioners and patients from safety-net clinics. Subst Abuse. 2017;38(2):213-221. doi:10.1080/ 08897077.2017.1296524
14. Manchikanti L, Kaye AM, Knezevic NN, et al. Responsible, safe, and effective prescription of opioids for chronic non-cancer pain: American Society of Interventional Pain Physicians (ASIPP) guidelines. Pain Physician. 2017;20(2s):S3-S92. doi:10.36076/ppj.2017.s92

15. Gourlay DL, Heit HA, Almahrezi A. Universal precautions in pain medicine: a rational approach to the treatment of chronic pain. Pain Med. 2005;6(2):107-112. doi:10.1111/j.1526-4637.2005.05031.x

16. Chou R, Ballantyne JC, Fanciullo GJ, Fine PG, Miaskowski C. Research gaps on use of opioids for chronic noncancer pain: findings from a review of the evidence for an American Pain Society and American Academy of Pain Medicine clinical practice guideline. J Pain. 2009;10(2):147-159. doi:10.1016/j.jpain.2008.10.007

17. Campbell G, Hall WD, Peacock A, et al. Effect of cannabis use in people with chronic non-cancer pain prescribed opioids: findings from a 4-year prospective cohort study. Lancet Public Health. 2018;3(7):e341-e350. doi:10.1016/S2468-2667(18)30110-5

18. Chou R, Fanciullo GJ, Fine PG, et al. Clinical guidelines for the use of chronic opioid therapy in chronic noncancer pain. J Pain. 2009;10 (2):113-130. e122. doi:10.1016/j.jpain.2008.10.008

19. Manchikanti L, Abdi S, Atluri S, et al. American Society of Interventional Pain Physicians (ASIPP) guidelines for responsible opioid prescribing in chronic non-cancer pain: part I-evidence assessment. Pain Physician. 2012;15(3 Suppl):S1-S65.

20. Manchikanti L, Abdi S, Atluri S, et al. American Society of Interventional Pain Physicians (ASIPP) guidelines for responsible opioid prescribing in chronic non-cancer pain: part 2-guidance. Pain Physician. 2012;15(3 Suppl):S67-116.

21. Trescot AM, Boswell MV, Atluri SL, et al. Opioid guidelines in the management of chronic non-cancer pain. Pain Physician. 2006;9 (1):1-39.

22. Max M. World Health Organization cancer pain relief program: network news. J Pain Symptom Manage. 1986;1(1):53-57. doi:10.1016/ S0885-3924(86)80035-5

23. Organization WH. Cancer Pain Relief: With a Guide to Opioid Availability. World Health Organization; 1996.

24. Ballantyne JC, Kalso E, Stannard C. WHO analgesic ladder: a good concept gone astray. BMJ (Clinical Research Ed). 2016;352:i20. doi:10.1136/bmj.i1717

25. Stjernswärd J. WHO cancer pain relief programme. Cancer Surv. 1988;7(1):195-208.

26. Hsu ES. Medication overuse in chronic pain. Curr Pain Headache Rep. 2017;21(1):2. doi:10.1007/s11916-017-0606-z

27. Breivik H. Opioids in cancer and chronic non-cancer pain therapy-indications and controversies. Acta Anaesthesiol Scand. 2001;45(9):1059-1066. doi:10.1034/j.1399-6576.2001.450902.x

28. Volkow ND, McLellan TA, Cotto JH, Karithanom M, Weiss SR. Characteristics of opioid prescriptions in 2009. JAMA. 2011;305 (13):1299-1301. doi:10.1001/jama.2011.401

29. Volkow ND, McLellan TA. Curtailing diversion and abuse of opioid analgesics without jeopardizing pain treatment. JAMA. 2011;305 (13):1346-1347. doi:10.1001/jama.2011.369

30. Jamison RN, Kerry Anne Sheehan B, Elizabeth Scanlan N, Ross EL. Beliefs and attitudes about opioid prescribing and chronic pain management: survey of primary care providers. J Opioid Manag. 2014;10 (6):375-382. doi:10.5055/jom.2014.0234

31. Mahan KT. The opioid crisis. J Foot Ankle Surg. 2017;56(1):1-2. doi:10.1053/j.jfas.2016.10.002

32. Jena AB, Goldman D, Weaver L, Karaca-Mandic P. Opioid prescribing by multiple providers in medicare: retrospective observational study of insurance claims. BMJ (Clinical Research Ed). 2014;348:g1393.

33. Rudd RA. Increases in drug and opioid-involved overdose deathsUnited States, 2010-2015. MMWR Morbiditymortal Weekly Rep. 2016;65.

34. Koenig KL. The opioid crisis in America: too much, too little, too late. West J Emerg Med. 2018;19(3):557-558. doi:10.5811/westjem.2018.2. 38087 
35. Sullivan MD, Edlund MJ, Steffick D, Unutzer J. Regular use of prescribed opioids: association with common psychiatric disorders. Pain. 2005;119(1-3):95-103. doi:10.1016/j.pain.2005.09.020

36. Rummans TA, Burton MC, Dawson N In Reply II-root causes of opioid crisis. Paper presented at: Mayo Clinic Proceedings; 2018.

37. Allen JD, Casavant MJ, Spiller HA, Chounthirath T, Hodges NL, Smith GA. Prescription opioid exposures among children and adolescents in the United States: 2000-2015. Pediatrics. 2017;139:4. doi:10.1542/peds.2016-3382

38. Yan E, Kuo DJ. I just need an opiate refill to get me through the weekend. J Med Ethics. 2019;45(4):219-224. doi:10.1136/medethics2018-105099

39. Bonica J. History of pain concepts and pain therapy. Mt Sinai J Med. 1991;58(3):191-202.

40. Dowell D, Haegerich TM, Chou R. CDC guideline for prescribing opioids for chronic pain-United States, 2016. JAMA. 2016;315 (15):1624-1645. doi:10.1001/jama.2016.1464

41. Management ASoATFoCP. Practice guidelines for chronic pain management: an updated report by the American Society of Anesthesiologists Task Force on Chronic Pain Management and the American Society of Regional Anesthesia and Pain Medicine. Anesthesiology. 2010;112 (4):810. doi:10.1097/ALN.0b013e3181c43103

42. Templeman K, Robinson A. Integrative medicine models in contemporary primary health care. Complement Ther Med. 2011;19 (2):84-92. doi:10.1016/j.ctim.2011.02.003

43. Madsen C, Vaughan M, Koehlmoos TP. Use of integrative medicine in the United States military health system. Evid Based Complement Alternat Med. 2017;2017:9529257. doi:10.1155/2017/9529257

44. Stanos SP. Stemming the tide of the pain and opioid crisis: AAPM reaffirms its commitment to multidisciplinary biopsychosocial care and training. Pain Med. 2017;18(6):1005-1006. doi:10.1093/pm/pnx120

45. Complementary NCf, Health I. Complementary, Alternative, or Integrative Health: What's in a Name? US Department of Health and Human Services, National Institutes of Health; 2015.

46. Vickers AJ, Vertosick EA, Lewith G, et al. Acupuncture for chronic pain: update of an individual patient data meta-analysis. J Pain. 2017;19(5):455-474.

47. Sarzi-Puttini P, Vellucci R, Zuccaro SM, Cherubino P, Labianca R, Fornasari D. The appropriate treatment of chronic pain. Clin Drug Investig. 2012;32(1):21-33. doi:10.2165/11630050-000000000-00000

48. Raffa RB, Pergolizzi JV Jr. A modern analgesics pain 'pyramid'. J Clin Pharm Ther. 2014;39(1):4-6. doi:10.1111/jcpt.2013.39.issue-1

49. Leung L. From ladder to platform: a new concept for pain management. J Prim Health Care. 2012;4(3):254-258. doi:10.1071/ $\mathrm{HC} 12258$
50. Cuomo A, Bimonte S, Forte CA, Botti G, Cascella M. Multimodal approaches and tailored therapies for pain management: the trolley analgesic model. J Pain Res. 2019;12:711-714. doi:10.2147/JPR. S178910

51. Riley J, Ross JR, Gretton SK, et al. Proposed 5-step World Health Organization analgesic and side effect ladder. Eur J Pain Suppl. 2007;1(S1):23-30. doi:10.1016/S1754-3207(08)60008-5

52. Lin YC, Wan L, Jamison RN. Using integrative medicine in pain management: an evaluation of current evidence. Anesth Analg. 2017;125(6):2081-2093. doi:10.1213/ANE.0000000000002579

53. Sharan AD, Riley J, Falowski S, et al. Association of opioid usage with spinal cord stimulation outcomes. Pain Med. 2018;19 (4):699-707. doi: $10.1093 / \mathrm{pm} / \mathrm{pn} \times 262$

54. Manchikanti L, Benyamin RM, Singh V, et al. An update of the systematic appraisal of the accuracy and utility of lumbar discography in chronic low back pain. Pain Physician. 2013;16(2 Suppl):SE55-SE95.

55. Berterame S, Erthal J, Thomas J, et al. Use of and barriers to access to opioid analgesics: a worldwide, regional, and national study. The Lancet. 2016;387(10028):1644-1656. doi:10.1016/S0140-6736(16)00161-6

56. Duthey B, Scholten W. Adequacy of opioid analgesic consumption at country, global, and regional levels in 2010, its relationship with development level, and changes compared with 2006. J Pain Symptom Manage. 2014;47(2):283-297. doi:10.1016/j.jpainsymman.2013.03.015

57. Huang Y. Current status of pain management in China: an overview. Eur J Pain. 2001;5(Suppl A):67-71. doi:10.1053/eujp.2001.0283

58. Xiao H, Liu H, Liu J, et al. Pain prevalence and pain management in a Chinese Hospital. Med Sci Monitor. 2018;24:7809-7819. doi:10.12659/ MSM.912273

59. Fang W, Liu T, Gu Z, Li Q, Luo C. Consumption trend and prescription pattern of opioid analgesics in China from 2006 to 2015. Eur J Hosp Pharm. 2019;26(3):140-145. doi:10.1136/ejhpharm-2017-001460

60. Yuan QL, Guo TM, Liu L, Sun F, Zhang YG. Traditional Chinese medicine for neck pain and low back pain: a systematic review and meta-analysis. PLoS One. 2015;10(2):e0117146. doi:10.1371/journal. pone. 0117146

61. Hui KK, Zhang WJ. China is poised to build a better healthcare delivery system using the integrative health paradigm. Zhongguo Zhong Xi Yi Jie He Xue hui. 2011;31(1):107-110.

62. Gu W. Update on administration of anesthetics and psychoactive drugs for pain management in China. Acta Anaesthesiologica Taiwanica. 2015;53(2):55-57. doi:10.1016/j.aat.2015.05.007

63. Yao J. How to establish and develop Pain Medicine Department? Pain Clin J. 2007;3(6):466.
Journal of Pain Research

\section{Publish your work in this journal}

The Journal of Pain Research is an international, peer reviewed, open access, online journal that welcomes laboratory and clinical findings in the fields of pain research and the prevention and management of pain. Original research, reviews, symposium reports, hypothesis formation and commentaries are all considered for publication. The manuscript

Submit your manuscript here: https://www.dovepress.com/journal-of-pain-research-journal management system is completely online and includes a very quick and fair peer-review system, which is all easy to use. Visit http:// www.dovepress.com/testimonials.php to read real quotes from published authors. 